Supporting Information

\title{
Multi-functional Nanomodulators Regulate Multiple Pathways to Enhance Antitumor Immunity
}

Yadan Zheng', Zhanzhan Zhangt, Qi Liut, Yu Zhao', Chunxiong Zheng', Jialei Hao', Kaikai Y's, Ying Wang', Chun Wangt, Xinzhi Zhao', Linqi Shi*t, Chunsheng Kang ${ }^{\star S}$ and Yang Liü

$\uparrow$ State Key Laboratory of Medicinal Chemical Biology, Key Laboratory of Functional Polymer Materials of Ministry of Education, College of Chemistry, Nankai University, National Demonstration Center for Experimental Chemistry Education, Nankai University, Tianjin, 300071, China. E-mail: shilinqi@nankai.edu.cn; yliu@nankai.edu.cn

$\S$ Tianjin Neurological Institute, Key Laboratory of Post-neurotrauma Neurorepair and Regeneration in Central Nervous System, Ministry of Education and Tianjin City, Department of Neurosurgery, Tianjin Medical University General Hospital, Tianjin 300052, China, E-mail: kang97061@tmu.edu.cn 


\section{Table of Contents}

Materials...

Instruments

Cell culture. S5

In vitro cytotoxicity analysis of MFNM

Pharmacokinetic studies of MFNM.

In vivo biodistribution of MFNM.

In vivo toxicity of MFNM .S6

Immunofluorescence staining .S6

Cytokine detection.

Statistical Analysis . $\mathrm{S} 7$

References S18 


\section{Materials}

2-(Hexamethyleneimino) ethanol (C7A), cetyltrimethylammonium chloride (CTAC), 1-ethyl-3-(3-dimethylaminopropyl)carbodiimide hydrochloride $(\mathrm{EDC} \cdot \mathrm{HCl}) \mathrm{N}$ hydroxysuccinimide (NHS) were purchased from Energy Chemical (Shanghai, China). Trimethylamine, tetraethoxysilane (TEOS, 98\%), $N$-(3-Aminopropyl)-methacrylamine hydrochloride (Apm), N, N'-methylene bisacryamide (Bis), ammonium persulfate (APS), tetramethylethylenediamine (TEMED) and sodium acetate trihydrate $\left(\mathrm{NaAc} \cdot 3 \mathrm{H}_{2} \mathrm{O}\right)$ were purchased from Aladdin (Tianjin, China). 3methacryloxypropyltrimethoxysilane, methacryloyl chloride, and 2methacryloyloxyethyl phosphorylcholine (MPC) were purchased from Heowns (Tianjin, China). phalloidin FITC, paraformaldehyde, and 4,6-diamidino-2phenylindole dihydrochloride (DAPI) were purchased from Invitrogen (USA). Cy5NHS and Cy3-NHS were obtained from Oukainasi Tech Inc. (Beijing, China). Antibody for flow cytometry and confocal laser scanning microscope observation (CLSM) were purchased from Invitrogen and Biolegend (USA). All ELISA kits were obtained from Elabscience (China). Mouse melanoma cells B16F10 was purchased from American Type Culture Collection (ATCC). Trypsin, fetal bovine serum (FBS) and RPMI 1640 culture medium were purchased from Sigma-Aldrich (Shanghai, China). THF, $\mathrm{HCl}$, methanol and other chemical reagents were purchased from local chemical companies.

\section{Instruments}

Dynamic light scattering (DLS) and zeta potential measurements were performed on a Brookheaven ZETAPALS/BI-200SM (Brookheaven Instrument, USA). Transmission electron microscopy (TEM) observations were performed on a Talos F200C electron microscope at an acceleration voltage of $120 \mathrm{kV}$. ${ }^{1} \mathrm{H}$ nuclear magnetic resonance $\left({ }^{1} \mathrm{H}\right.$ NMR) spectra were obtained on a Varian UNITY-plus 400 M NMR spectrometer. Fluorescence spectra were measured on Hitachi F4600. UV-Visible spectra were acquired with a NanoDrop One ${ }^{\mathrm{c}}$ (Thermo Scientific, USA). Ultraviolet absorption was measured on a Tecan Spark plate reader. Flow cytometry analysis was performed on a 
Guava easyCyte 8HT flow cytometry. Confocal laser scanning microscope (CLSM) images were captured on a FluoView Confocal Laser Scanning Microscopes-FV1000.

\section{Synthesis of 2-(Hexamethyleneimino)ethyl methacrylate monomers}

Synthesis of 2-(Hexamethyleneimino)ethyl methacrylate (C7A-MA) is described as a representative procedure. ${ }^{1}$ First, 2-(pentamethyleneimino)ethanol (14.3 g, $0.1 \mathrm{~mol}$ ), triethylamine $(10.1 \mathrm{~g}, 0.1 \mathrm{~mol})$, and hydroquinone $(0.11 \mathrm{~g}, 0.001 \mathrm{~mol})$ were dissolved in $100 \mathrm{~mL}$ THF. Methacryloyl chloride (10.4 g, $0.1 \mathrm{~mol})$ was then added dropwise into a three-neck flask within $30 \mathrm{~min}$ at $0^{\circ} \mathrm{C}$. The reaction mixture was stirred at room temperature overnight. After the reaction, the mixture was filtered to remove the precipitate. After the solvent was removed under reduced pressure, the residue was purified by silica gel chromatography (light petroleum/ethyl acetate $=5 / 1, \mathrm{v} / \mathrm{v}$ ) to yield C7A-MA as a yellow liquid. Yield: $68 \%$.

${ }^{1} \mathrm{H}$ NMR (400 MHz, $\left.\mathrm{CDCl}_{3}, 25{ }^{\circ} \mathrm{C}, \mathrm{TMS}, \delta\right): 6.09$ (br, $\left.1 \mathrm{H}, \mathrm{CH} H=\mathrm{C}\left(\mathrm{CH}_{3}\right)-\right), 5.55$ (br, $\left.1 \mathrm{H}, \mathrm{CH} H=\mathrm{C}\left(\mathrm{CH}_{3}\right)-\right), 4.24\left(\mathrm{t}, J=6.5 \mathrm{~Hz}, 2 \mathrm{H},-\mathrm{OCH}_{2} \mathrm{CH}_{2} \mathrm{~N}-\right), 2.84(\mathrm{t}, J=6.5 \mathrm{~Hz}, 2 \mathrm{H},-$ $\left.\mathrm{OCH}_{2} \mathrm{CH}_{2} \mathrm{~N}-\right), 2.72\left(\mathrm{~m}, 4 \mathrm{H},-\mathrm{N}\left(\mathrm{CH}_{2} \mathrm{CH}_{2} \mathrm{CH}_{2}\right)_{2}\right), 1.94$ (s, 3H, $\left.\mathrm{CH}_{2}=\mathrm{C}\left(\mathrm{CH}_{3}\right)-\right), 1.63$ (m, $\left.4 \mathrm{H},-\mathrm{N}\left(\mathrm{CH}_{2} \mathrm{CH}_{2} \mathrm{CH}_{2}\right)_{2}\right), 1.58\left(\mathrm{~m}, 4 \mathrm{H},-\mathrm{N}\left(\mathrm{CH}_{2} \mathrm{CH}_{2} \mathrm{CH}_{2}\right)_{2}\right)$ (Figure $\mathrm{S} 2$ ).

\section{Synthesis of MSN}

In a typical synthesis, ${ }^{2} 53.4 \mathrm{~g}$ of DI-water, $6.24 \mathrm{~g}$ of CTAC, and $0.3 \mathrm{~g}$ of $\mathrm{NaAc} \cdot 3 \mathrm{H}_{2} \mathrm{O}$ were mixed and stirred at temperature of $40^{\circ} \mathrm{C}$ for $2 \mathrm{~h}$. Then $4.35 \mathrm{~mL}$ of TEOS was added into the mixture dropwise within 3 min under steadily stirring at 500 RPM. The solution was stirred for $24 \mathrm{~h}$. After cooling to room temperature, the solution was centrifuged at 13,000 RPM for $25 \mathrm{~min}$ to isolate the products from the suspension. After washing with water for 3 times, the products were refluxed for $3 \mathrm{~h}$ with a $1 \mathrm{wt} \%$ solution of sodium chloride $(\mathrm{NaCl})$ in methanol at room temperature to remove the CTAC. Then the obtained MSN (100 mg) suspended in ethanol was reacted with 3methacryloxypropyltrimethoxysilane $(0.5 \mathrm{~mL})$ at room temperature in the dark overnight. The obtained nanoparticles were separated through centrifugation, washed several times with ethanol. 


\section{Cell culture}

B16F 10 cells and NIH 3T3 cells were obtained from American Type Culture Collection (ATCC) and cultured in RPMI 1640 with 10\% (v/v) fetal bovine serum (FBS), $100 \mathrm{U}$ $\mathrm{mL}^{-1}$ penicillin and $100 \mathrm{mg} \mathrm{mL}^{-1}$ streptomycin in an incubator (Thermo Scientific) at $37{ }^{\circ} \mathrm{C}$ under an atmosphere of $5 \% \mathrm{CO}_{2}$ and $90 \%$ relative humidity, respectively. The cells were sub-cultivated approximately every 2 days at $80 \%$ confluence using $0.25 \%$ $(\mathrm{w} / \mathrm{v})$ trypsin at a split ratio of 1:5.

\section{In vitro cytotoxicity analysis of MFNM}

The cytotoxicity of PM and MFNM were determined using CCK-8 viability assay. Briefly, cells (NIH 3T3 and B16F10) were seeded in 96-well plates at $5 \times 10^{3}$ cells per well and grown to $80 \%$ confluence, followed by replacing the culture medium with the fresh ones containing different nanoparticles at varied concentrations for further $24 \mathrm{~h}$ incubation. CCK-8 was mixed with RPMI 1640 at a volume ratio of 1/9 to achieve the CCK-8 working solution. After the incubation, the cells were rinsed with PBS, followed by the addition of $100 \mu \mathrm{L}$ CCK-8 working solution for additional $2 \mathrm{~h}$ incubation. Quantification of the cell viability was achieved by measuring the absorbance with Tecan's Infinite M200 microplate reader $(\lambda=450 \mathrm{~nm})$. The cell viability was calculated by referring to the absorbance of the cells without any treatment.

\section{Pharmacokinetic studies of MFNM}

To investigate the pharmacokinetic of MFNM, female C57BL/6J (6 8 weeks of age) mice were purchased from SPF Experimental Animal Technology Co., Ltd. All experimental protocols were conducted within Nankai University guidelines for animal research and were approved by Institutional Animal Care and Use Committee. The mice were injected intravenously with Cy5 labeled nanoparticles, following by collecting the blood samples $(40 \mu \mathrm{L})$ at different times. The concentration of MFNM in blood was quantitatively examined by detecting the fluorescent intensity at $670 \mathrm{~nm}$ excited at 633 $\mathrm{nm}$. 


\section{In vivo biodistribution of MFNM}

The tumor-bearing mice were generated by subcutaneous injection of B16F10 cells $\left(5 \times 10^{5}\right.$ for each mouse) in the right leg of C57BL/ 6 mice at 6 weeks old. The mice were randomly divided into different groups. When the tumor volume was about $250 \mathrm{~mm}^{3}$, the mice were intravenously injected with $100 \mu \mathrm{L}$ of PBS, Cy5-nonPMPC-NP, Cy5MFNM. At $24 \mathrm{~h}$ post-injection, the mice were sacrificed, and the major organs and the tumors were collected for ex vivo imaging. Ex vivo images were taken by IVIS Lumina imaged system (Caliper Life Sciences, USA). The fluorescence images were analyzed using Living Image 3.1 (Caliper Life Sciences, USA).

\section{In vivo toxicity of MFNM}

Female C57B6/L mice at 6-week old were administrated intravenously with PBS and MFNM for 7 days through tail vein. At the end of experiment, the mice from each group were sacrificed, and the blood were collected for blood chemistry analysis and blood routine analysis. The tests were performed using auto biochemistry analyzer and auto hematology analyzer, respectively. Major organs of the mice, including the hearts, the livers, the spleens, the lungs and the kidneys were collected, subsequently preserved in $4 \%$ buffered formaldehyde and embedded in paraffin. The paraffin sections were stained for examining the organ damage using H\&E staining.

\section{Immunofluorescence staining}

For immunofluorescence studies, tumor tissues were immersed in $4 \%$ buffered formalin phosphate solution for $24 \mathrm{~h}$, and then transferred to sucrose solution for dehydration. The tissues were then embedded in optimal cutting medium (O.C.T.), and frozen slices were made on a cryostat microtome. Immunofluorescence staining was performed by rinsing the slices with PBS, permeabilization, and blocking in 5\% BSA solution at room temperature for $1 \mathrm{~h}$. For the phenotype staining of $\mathrm{T}$ cells and Tregs, the fixed tumor sections were incubated with primary antibodies of CD4 (rat), CD8 (rabbit) and Foxp3 (rat), following by incubating with Alexa Fluor 488 conjugated second antibody and Alexa Fluor 594 conjugated second antibody. DAPI was used for nuclear 
counterstaining. CLSM images were captured on an Olympus CLSM with a 20× emersion objective.

\section{Cytokine detection}

The intratumor levels of TGF- $\beta$, IFN- $\gamma$, TNF- $\alpha$ and IL-12 were measured with ELISA kits according to the manufacturer's instructions. All of these experiments were performed in triplicate.

\section{Statistical Analysis}

Statistical comparisons were achieved using one-way ANOVA with Dunnett post-test with GraphPad Prism 7.0. Data represent mean \pm standard error of the mean (s.d.) deviation from at least three independent experiments $(n \geq 3)$ and the significance levels are $* \mathrm{p}<0.05, * * \mathrm{p}<0.01, * * * \mathrm{p}<0.001$ and $* * * * \mathrm{p}<0.0001$ 


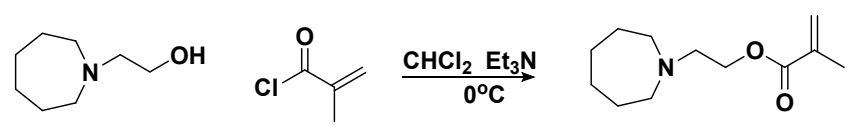

b
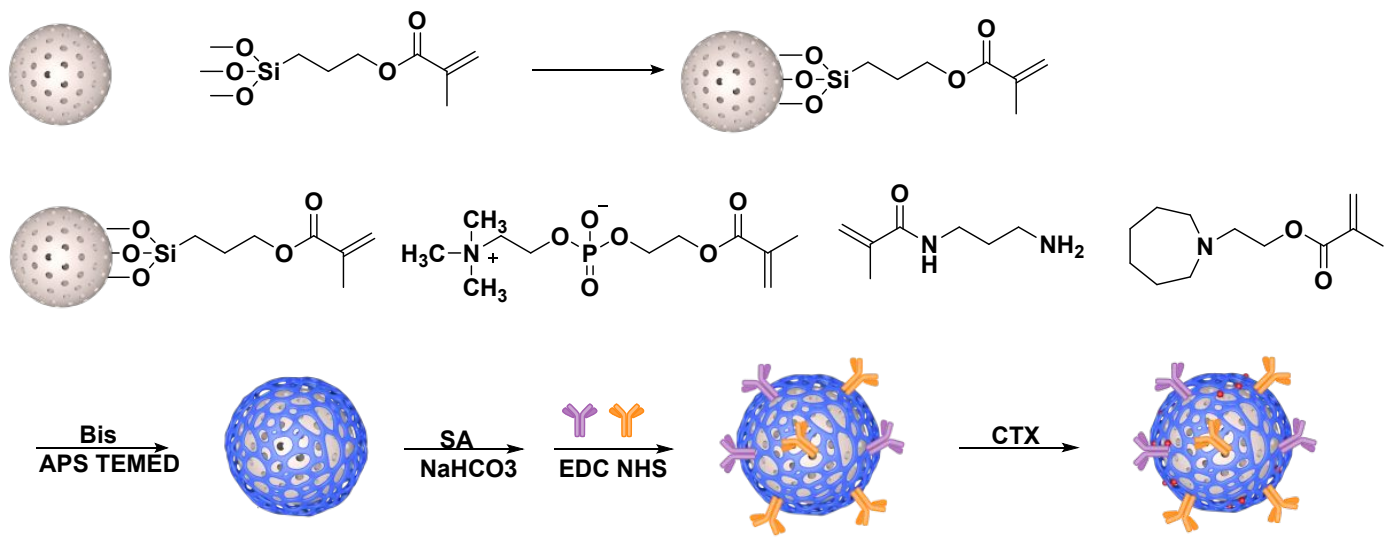

Figure S1. a) Synthesis routes of C7A-MA and b) schematic illustration of the MFNM synthesis.
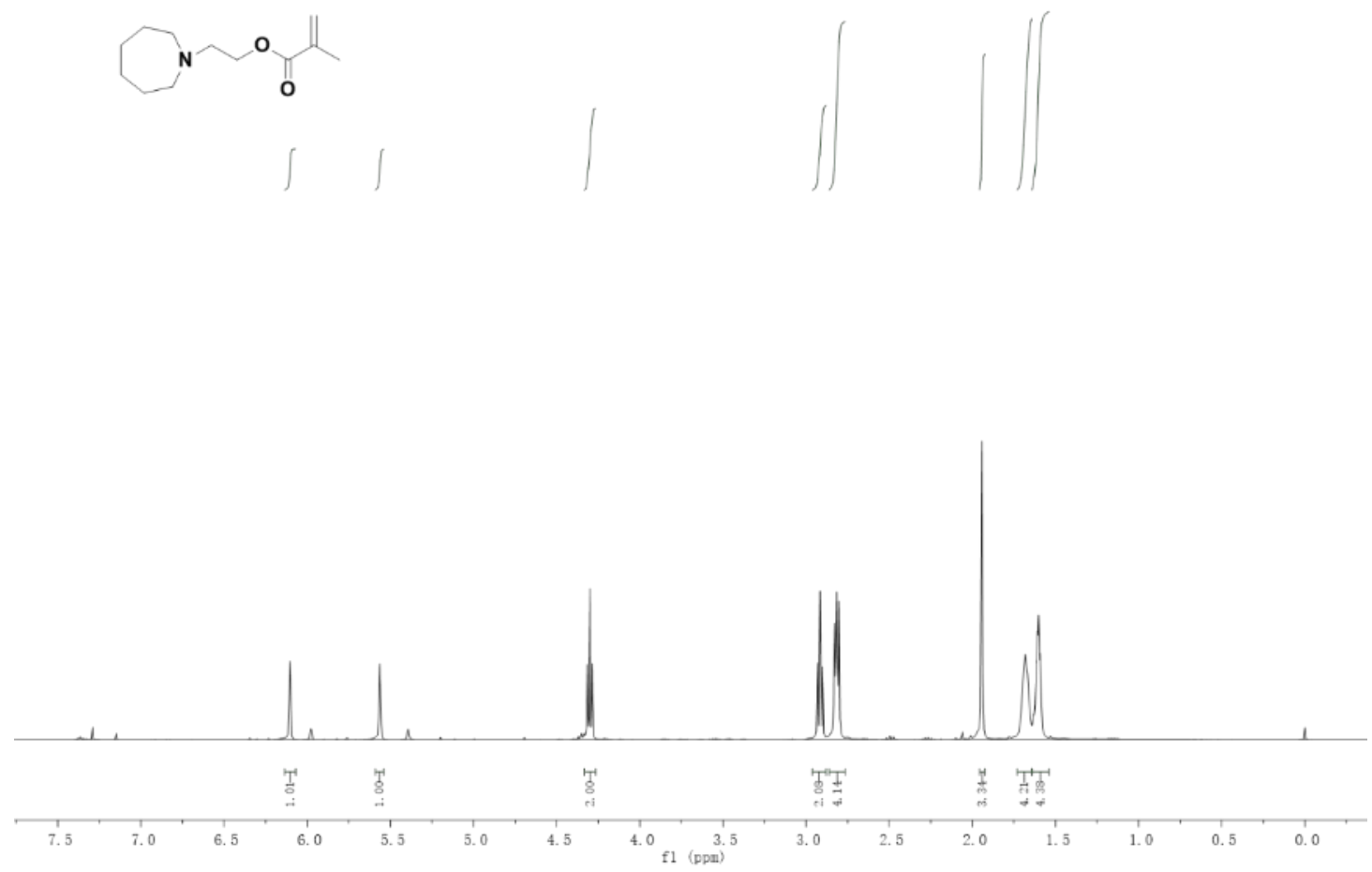

Figure S2. ${ }^{1} \mathrm{H}$ NMR spectra of 2-(Hexamethyleneimino) ethyl methacrylate (C7A-MA) in $\mathrm{CDCl}_{3}$. 


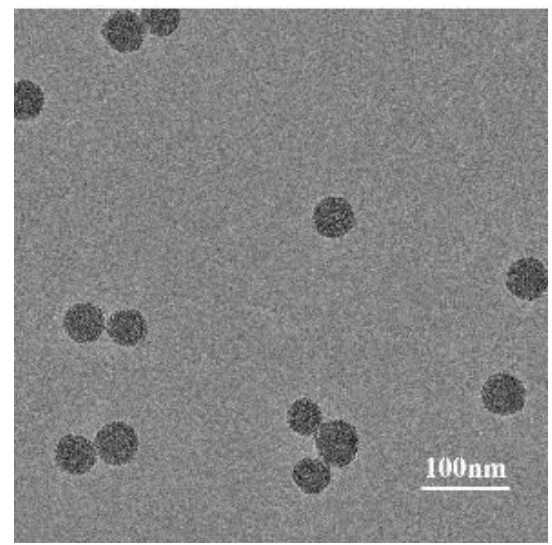

Figure S3. Morphology assessment of MSN using transmission electron microscopy (TEM) bright field. The scale bar represents $100 \mathrm{~nm}$.

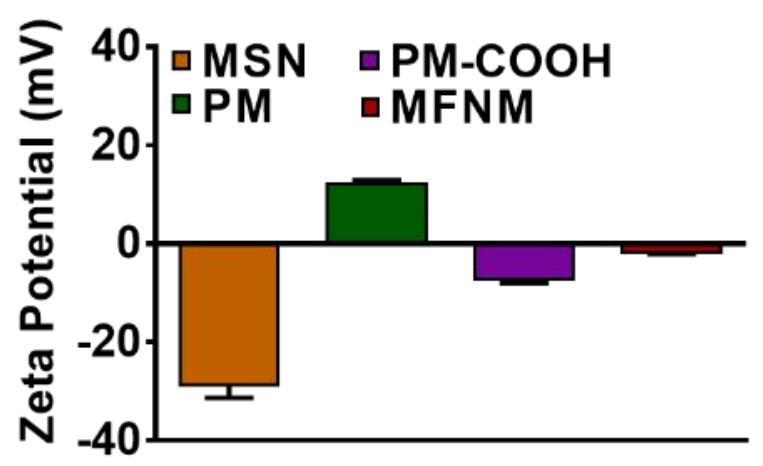

Figure S4. Zeta potential of MSN, PM, PM-COOH and MFNM. Data represent mean \pm standard derivations (s.d.) from three independent experiments $(n=3)$.

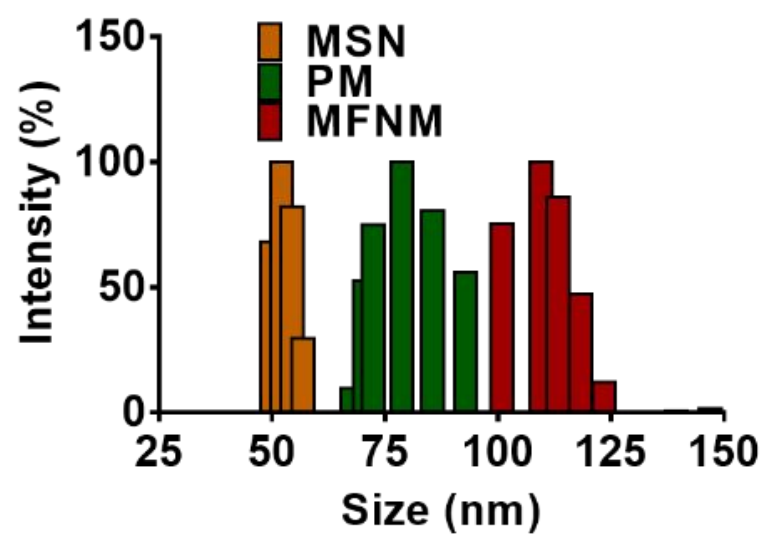

Figure S5. Hydrodynamic size distributions of MSN, PM, MFNM measured using dynamic light scattering (DLS). 


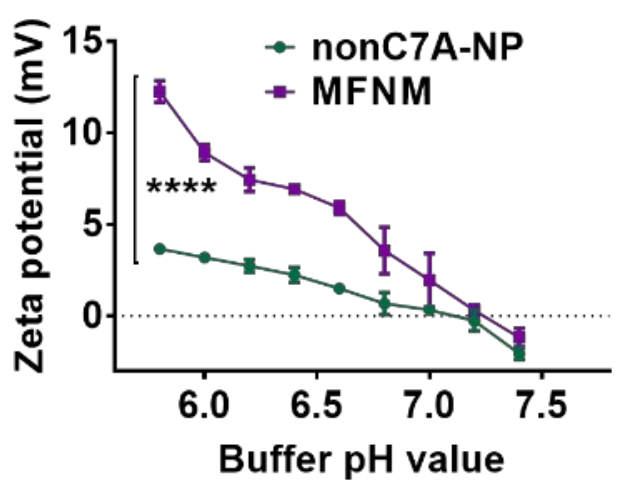

Figure S6. Zeta potential of nonC7A-NP and MFNM at different $\mathrm{pH}$ condition. Data represent mean $\pm \mathrm{s}$. d. from three independent experiments $(n=3)$.
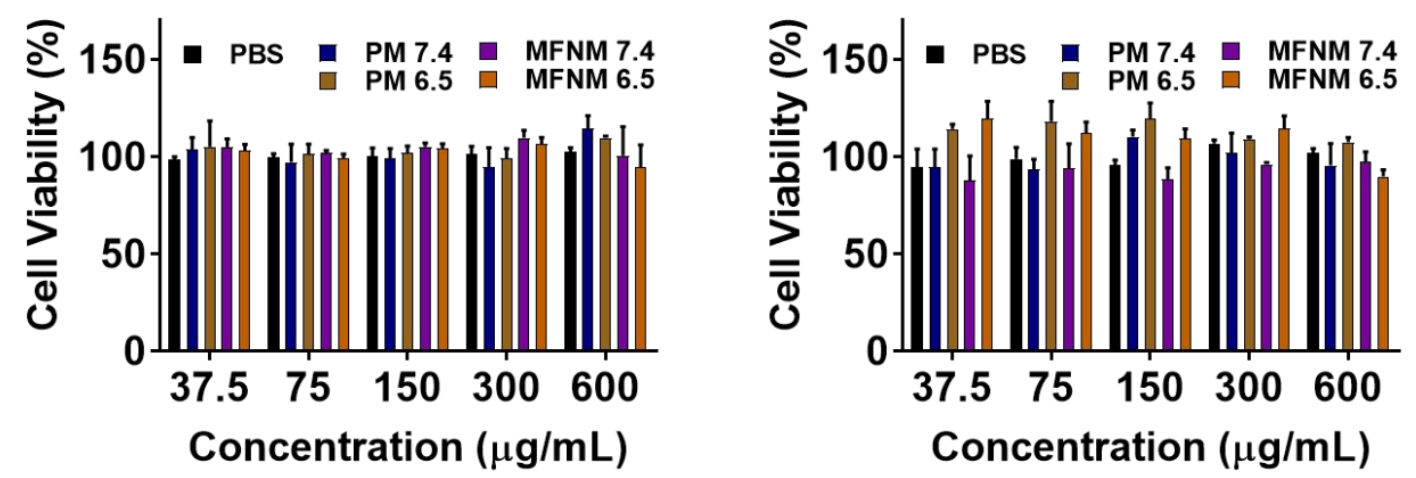

Figure S7. Cell viability of NIH 3 T3 cells and B16F10 cells after treating with PM and MFNM at $\mathrm{pH} 7.4$ and $\mathrm{pH} 6.5$ for $24 \mathrm{~h}$ incubation. Cell viability was assessed using CCK-8 assay. All data are presented as mean \pm s.d. from three independent experiments $(n=3)$. 


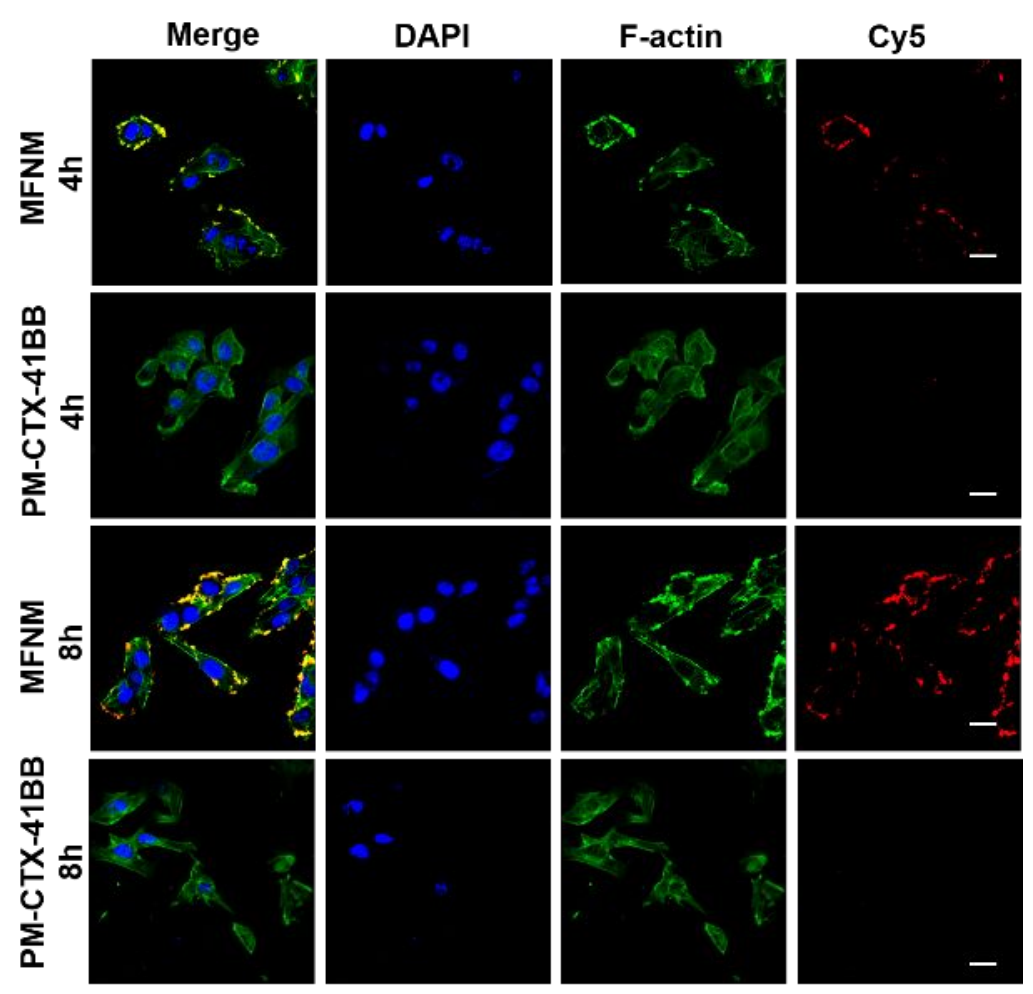

Figure S8. Confocal laser scanning microscope (CLSM) images of B16F10 cells after $4 \mathrm{~h}$ and $8 \mathrm{~h}$ incubation with the Cy5-labeled MFNM and the Cy5(red)-labeled PM-CTX41BB. Cellular nuclei and cytoskeleton F-actin were stained with DAPI (blue) and Phalloidin FITC (green), respectively. The scale bar represents $40 \mu \mathrm{m}$.

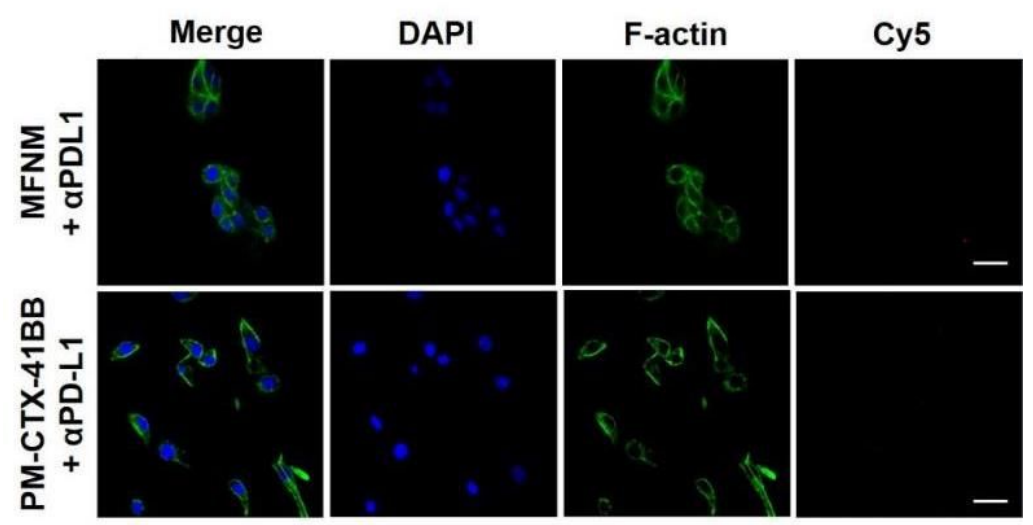

Figure S9. Confocal laser scanning microscope images (CLSM) of B16F10 cells treated with anti-PD-L1 mAbs prior to an incubation with the Cy5(red)-labeled PMCTX-41BB and MFNM. Cells were stained with DAPI (blue) and Phalloidin FITC (green). The scale bar represents $20 \mu \mathrm{m}$. 


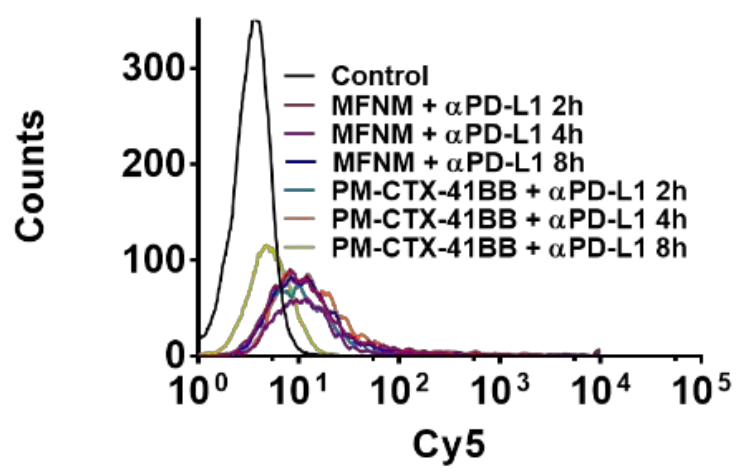

Figure S10. Flow cytometry profiles of B16F10 tumor cells pre-treated with anti-PDL1 mAbs prior to an incubation with Cy5(red)-labeled PM-CTX-41BB, MFNM at 37 ${ }^{\circ} \mathrm{C}$ for $2 \mathrm{~h}, 4 \mathrm{~h}$ and $8 \mathrm{~h}$.

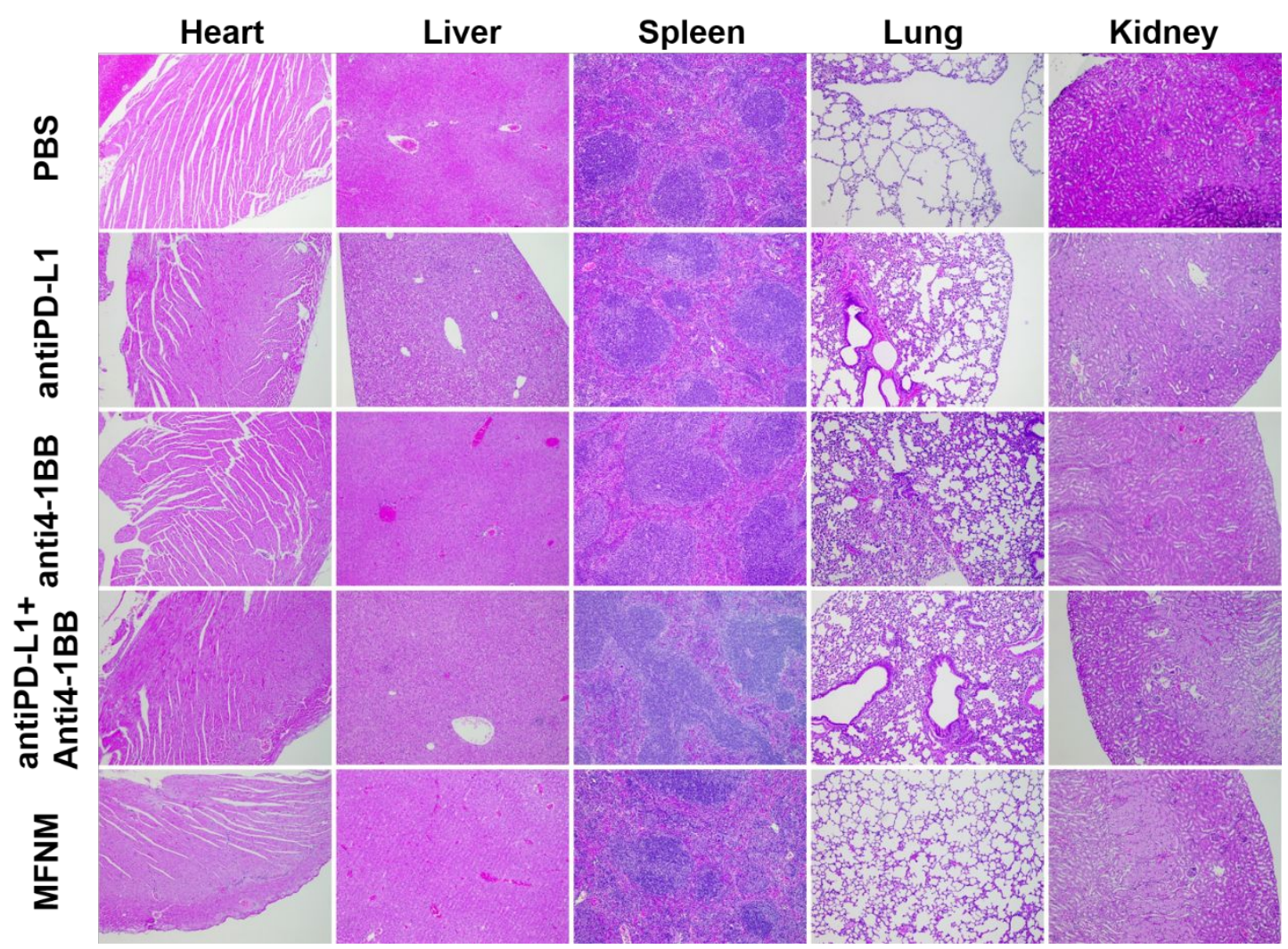

Figure S11. H\&E staining tissue sections from the heart, the liver, the spleen, the lung and the kidney from the mice with PBS and MFNM. 


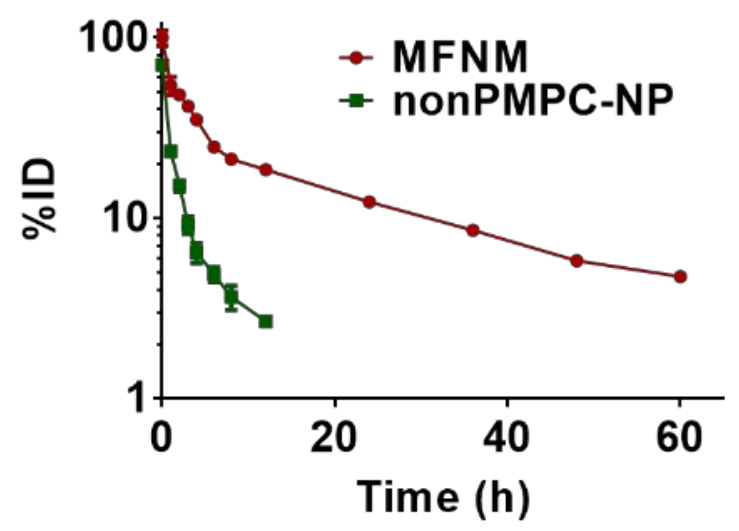

Figure S12. Pharmacokinetic curves of nanoparticles in mice after tail vein injection. Nanoparticles were pre-labelled with Cy5. Data represent mean \pm s.d. from three independent experiments $(n=3)$.
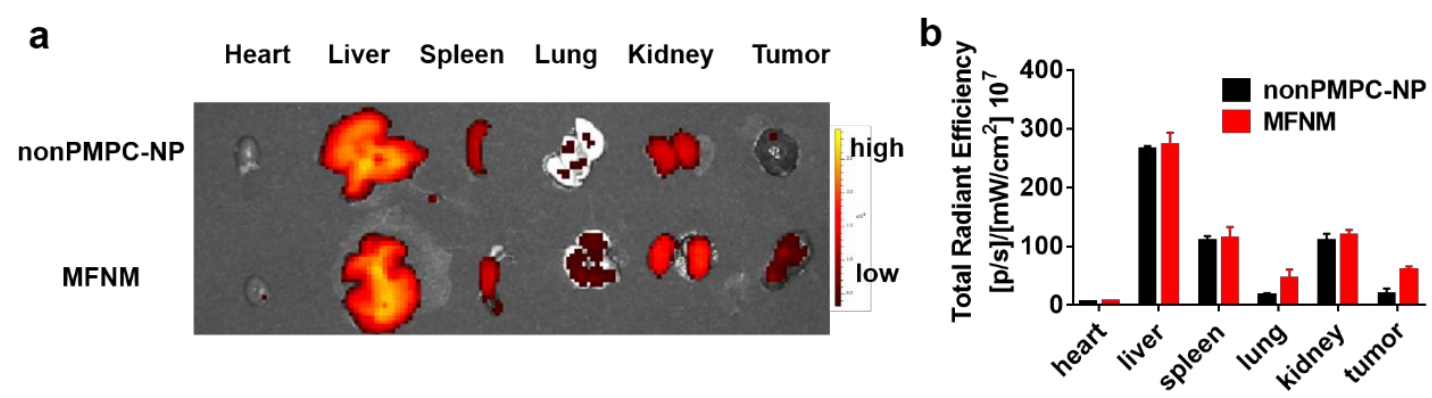

Figure S13. a) Ex vivo fluorescence imaging of the tumor and normal tissues harvested from the euthanized B16F10 tumor-bearing mice at $24 \mathrm{~h}$ post injection. b) Quantitative analysis of organ accumulation of nonPMPC-NP and MFNM based on the fluorescence intensity from the ex vivo images. Data represent mean \pm s.d. from three independent experiments $(n=3)$.

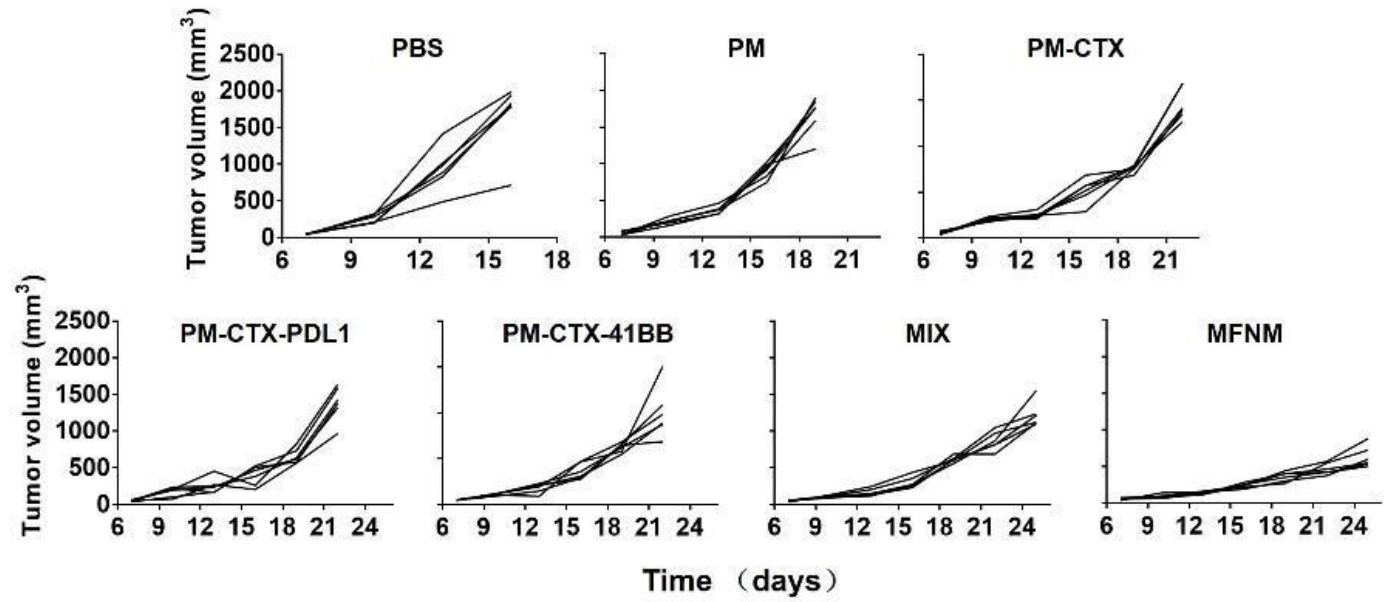


Figure S14. Individual tumor growth kinetics in different groups. Growth curves were stopped when the first mouse of the corresponding group died. Data represent mean \pm s.d. from six independent experiments $(n=6)$

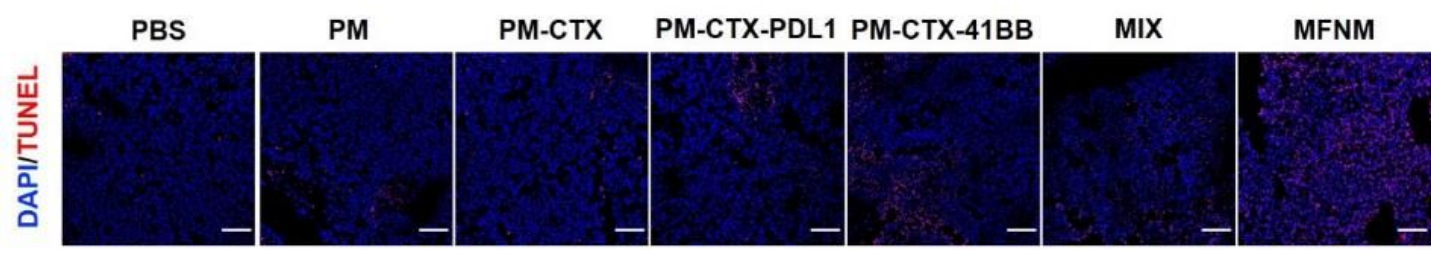

Figure S15. TUNEL analysis from the mice in tumor tissues treated with PBS, PM, PM-CTX, PM-CTX-41BB, PM-CTX-PDL1, MIX and MFNM. The scale bar represents $100 \mu \mathrm{m}$.

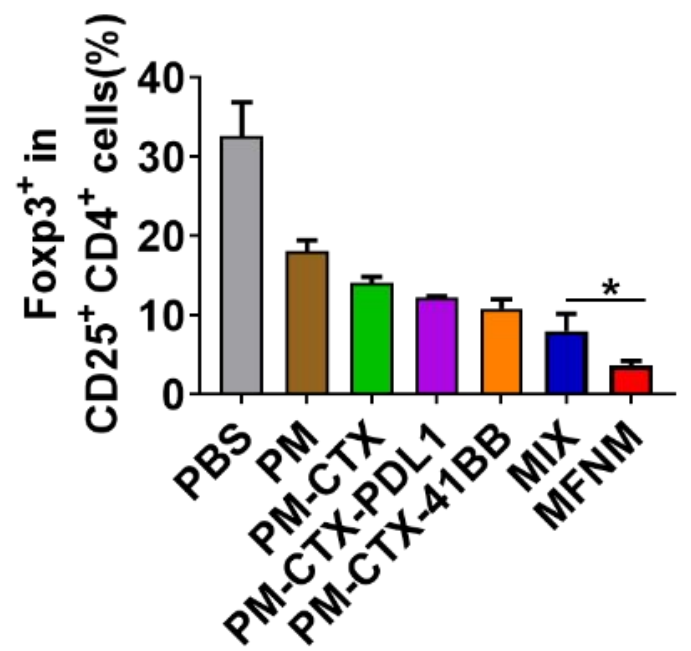

Figure S16. Flow cytometric analysis of the Tregs population within the tumor (gating on $\mathrm{CD} 45^{+} \mathrm{CD}^{+}$) at day 10 post-treatment. Data are presented as mean \pm s.d. from three independent experiments $(n=3)$.

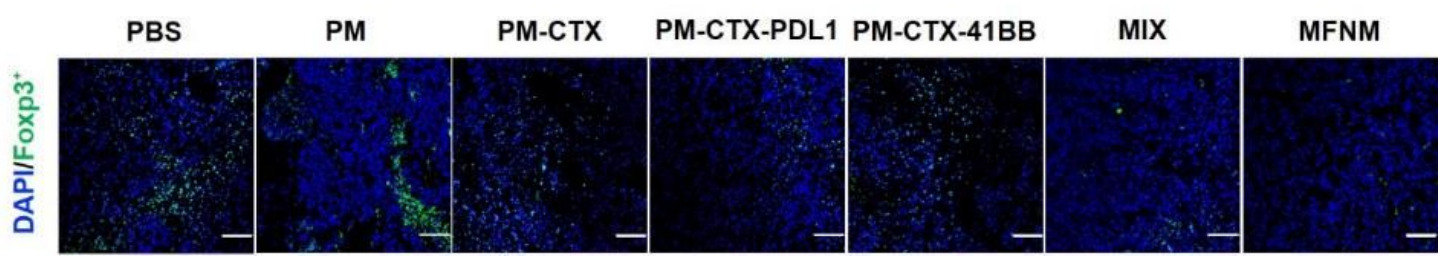

Figure S17. Representative immunofluorescence images of tumors showing Tregs infiltration in tumor tissues treated with PBS, PM, PM-CTX, PM-CTX-41BB, PMCTX-PDL1, MIX and MFNM. The scale bar represents $100 \mu \mathrm{m}$. 
a

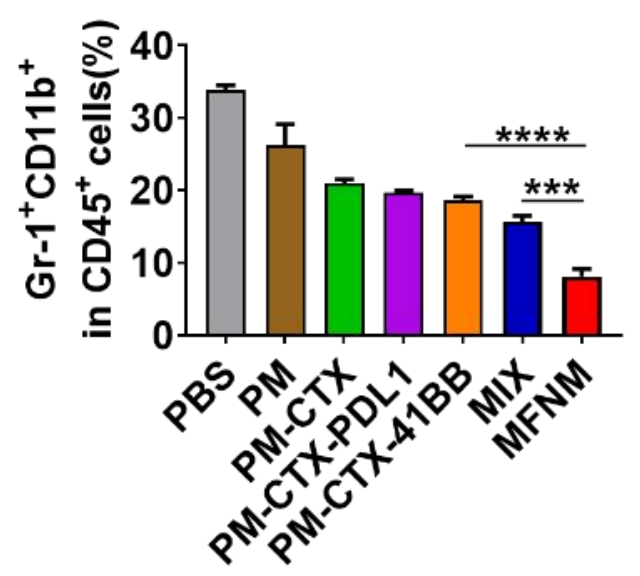

b

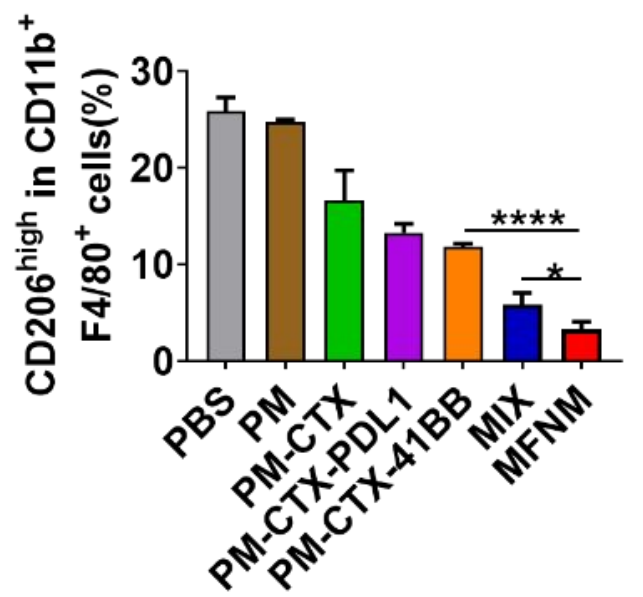

Figure S18. Flow cytometric analysis of MDSC a) infiltration within the tumor and the TAM b) infiltration within the tumor at day 10 post-treatment. Data are presented as mean \pm s.d. from three independent experiments $(n=3)$.

a

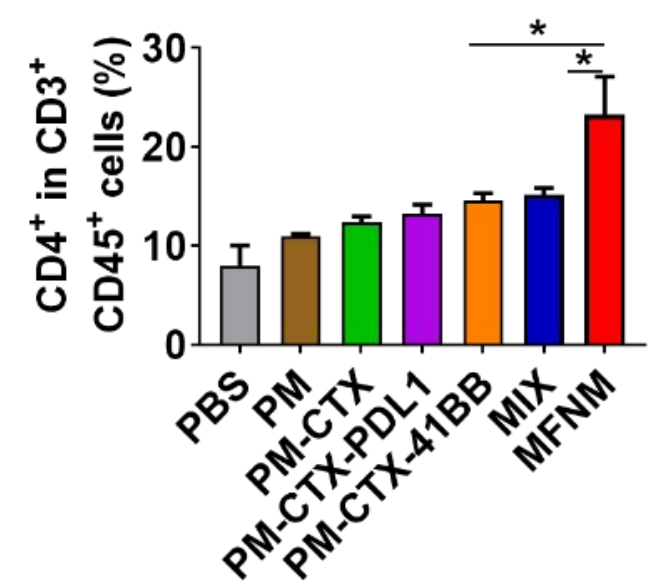

b

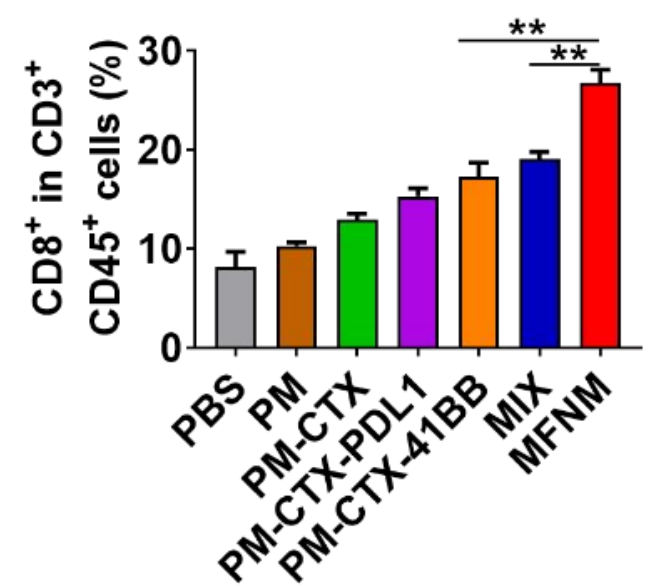

Figure S19. Flow cytometry analysis of the population of $\mathrm{CD} 4^{+} \mathrm{T}$ cells a) and $\mathrm{CD} 8^{+} \mathrm{T}$ cells b) within the tumor at day 10 post-treatment. Data are presented as mean \pm s.d. from three independent experiments $(n=3)$. 
Table S1. Optimization of MFNM conjugation chemistry

\begin{tabular}{|c|c|c|c|c|c|}
\hline \multirow{2}{*}{ Sample } & \multirow{2}{*}{$\begin{array}{l}\text { Incubation }(\mu \mathrm{g} / \mathrm{mg}) \\
{[\alpha P D L 1]:[\alpha 41 \mathrm{BB}]}\end{array}$} & \multirow{2}{*}{$\begin{array}{c}\text { Feeding } \\
\text { Ratio }\end{array}$} & \multicolumn{2}{|c|}{$[$ Conjugation] $(\mu \mathrm{g} / \mathrm{mg})$} & \multirow{2}{*}{$\begin{array}{c}\text { Conjugation } \\
\text { Ratio }\end{array}$} \\
\hline & & & [aPDL1] & [41BB] & \\
\hline 1 & 100:100 & $1: 1$ & 33.50 & 30.67 & 1.09 \\
\hline 2 & $200: 100$ & $2: 1$ & 29.63 & 35.70 & 0.83 \\
\hline 3 & $100: 200$ & $1: 1$ & 27.75 & 27.07 & 1.01 \\
\hline 4 & $200: 200$ & $2: 2$ & 31.63 & 34.47 & 0.92 \\
\hline
\end{tabular}

Table S2. Blood routine analysis in blood samples of C57BL/6 mice on 7 days after treatment

\begin{tabular}{ccccc}
\hline Group & WBC & RBC & PLT & HGB \\
& $\mathbf{( 1 0} / \mathbf{L})$ & $\mathbf{( 1 0 ^ { 1 2 } / \mathbf { L } )}$ & $\mathbf{( 1 0 9 / \mathbf { L } )}$ & $\mathbf{( g / L )}$ \\
\hline PBS & $4.6 \pm 0.14$ & $8.2 \pm 0.33$ & $1060 \pm 42.4$ & $131.5 \pm 6.4$ \\
antiPD-L1 & $6.5 \pm 0.6$ & $8.7 \pm 1.4$ & $692 \pm 223.4$ & $135.5 \pm 21.9$ \\
anti4-1BB & $3.33 \pm 0.8$ & $8.4 \pm 1.7$ & $625.3 \pm 126.9$ & $127.7 \pm 29.8$ \\
antiPD-L1 + & $1.9 \pm 0.55$ & $6.5 \pm 0.5$ & $398.1 \pm 74.2$ & $97.3 \pm 7.5$ \\
anti4-1BB & & & & \\
MFNM & $6.9 \pm 3.5$ & $7.3 \pm 1.9$ & $810 \pm 135.1$ & $114 \pm 21.7$ \\
\hline
\end{tabular}


Table S3. Values of serum enzymes in blood samples of C57BL/6 mice on 7 days

\begin{tabular}{ccccc}
\multicolumn{5}{c}{ after treatment } \\
Group & AST & ALP & ALT & BUN \\
& $(\mathbf{U} / \mathbf{L})$ & $(\mathbf{U} / \mathbf{L})$ & $(\mathbf{U} / \mathbf{L})$ & $(\mathbf{m m o l} / \mathbf{L})$ \\
\hline PBS & $59.5 \pm 6.15$ & $153.7 \pm 7.63$ & $293.35 \pm 4.158$ & $10.43 \pm 0.74$ \\
antiPD-L1 & $60.15 \pm 13.3$ & $209.9 \pm 18.5$ & $209.6 \pm 47.8$ & $8.48 \pm 0.94$ \\
anti4-1BB & $71.7 \pm 9.34$ & $172.5 \pm 18.3$ & $226.17 \pm 26.8$ & $11.25 \pm 1.13$ \\
antiPD-L1 + & $71.7 \pm 6.8$ & $282.47 \pm 13.9$ & $168.08 \pm 5.54$ & $8.17 \pm 1.35$ \\
anti4-1BB & & & & \\
MFNM & $67.15 \pm 1.48$ & $177.87 \pm 16.9$ & $274.65 \pm 14.78$ & $10.39 \pm 1.5$ \\
\hline
\end{tabular}

Table S4. Comparison of the Intravenous Pharmacokinetic Parameters for nonPMPCNP and MFNM.

\begin{tabular}{ccc}
\hline Parameters & nonPMPC-NP & MFNM \\
\hline $\mathbf{t}_{\mathbf{1} / \mathbf{2}}(\mathbf{h})$ & 2.897 & 0.7027 \\
$\mathbf{A U C}(\mathbf{U} / \mathbf{L})$ & 857 & 135 \\
$\mathbf{A M U C}(\mathbf{U} / \mathbf{L} \mathbf{h})$ & 15355 & 5233 \\
$\mathbf{C L}(\mathbf{m L} / \mathbf{h})$ & 1.078 & 35.74 \\
$\mathbf{V}_{\mathbf{d}}(\mathbf{m L})$ & 0.003 & 6.24 \\
MRT (h) & 17.917 & 3.876 \\
\hline
\end{tabular}




\section{References}

(1) Zhou, K.; Wang, Y.; Huang, X.; Luby-Phelps, K.; Sumer, B. D.; Gao, J., Tunable, ultrasensitive $\mathrm{pH}$-responsive nanoparticles targeting specific endocytic organelles in living cells. Angew Chem. Int. Ed. Engl. 2011, 50 (27), 6109-6114.

(2) Yu, M.; Zhou, L.; Zhang, J.; Yuan, P.; Thorn, P.; Gu, W.; Yu, C., A simple approach to prepare monodisperse mesoporous silica nanospheres with adjustable sizes. J. Colloid Interface Sci. 2012, 376 (1), 67-75. 